\title{
Wolfgang Wildgen
}

\section{Synergetische Modelle in der Soziolinguistik}

\author{
Zur Dynamik des Sprachwechsels Niederdeutsch-Hochdeutsch in \\ Bremen um die Jahrhundertwende (1880-1920)
}

Dieser Aufsatz verfolgt zwëi Ziele: Erstens soll exemplarisch gezeigt werden, in welcher Weise Konzeptionen und Modellbildungsverfahren der Synergetik in der Soziolinguistik zur Anwendung kommen können. Zweitens wird versucht, auf der Basis einer größeren Menge empirischer Fakten, die nicht modellspezifisch sind, ein qualitatives Verständnis des Sprachwechsels in Bremen, seiner Momente und Auslöser anzubahnen. In der Spannung zwischen informeller Rekonstruktion und synergetischem Modell können die Leistungen und Grenzen des vorgestellten Instrumentariums realistisch beurteilt werden. Exemplarisch vorgeführt wird außerdem der Übergang von der qualitativen Dynamik (in Abschnitt 2.3.) zur statistischen Dynamik (in Kapitel 3).*

\section{Synergetik und die dynamische Tradition in der Sprachgeographie}

Für unser Anwendungsfeld, die Sprachgeographie und, genereller, die Soziolinguistik, können wir die synergetischen Modelle durch drei zentrale Eigenschaften charakterisieren:

(1) In der langen Tradition der naturwissenschaftlichen Dynamik stehend, arbeitet die Synergetik wesentlich mit Methoden der Analysis und der Stabilitätstheorie. Sie enthält allerdings auch immer einen statistischen Theorieanteil, was sie von der Katastrophentheorie deutlich abhebt (vgl. Wildgen 1982, 1985).

* Unmittelbarer Anlaß für den Versuch einer synergetischen Modellbildung war die Kooperation mit Prof. Dr. Hermann Haken, Prof. Dr. Thomas T Ballmer und Dr. HansJürgen Eikmeyer bei der Vorbereitung der Eröffnungssitzung der 6. Jahrestagung der DGfS in Bielefeld 1984. Es fanden mehrere Arbeitstreffen in Stuttgart statt, bei denen ich meine Konzeption vortragen konnte. Eine kürzere Fassung dieser Arbeit lag meinem Korreferat zum Vortrag von Prof. Haken: „Ist die Sprache ein synergetisches System?“ bei der Eröffnung der Jahrestagung am 27.2.1984 zugrunde. Die Idee und Initiative zur Verbindung von Linguistik und Synergetik stammt von Thomas T Ballmer. Sein Tod kam wie ein plötzlicher Frost im Frühling für unser noch zaghaftes Unternehmen. Ihm sei diese Arbeit gewidmet. 
(2) Ihre Modelle vermitteln zwischen Mikro- und Makrobereich, in unserem Bcispiel zwischen individuellen Verhaltensweisen und Einstellungen einerscits und sozialen Strukturen sowie Veränderungen auf der Makroebene der Gesellschaft andererseits.

(3) Die Modelle verbinden dynamische Muster, etwa im Zusammenhang vom Übcrgang zu stabilen stationären Zuständen oder zu Grenzzyklen des Systcms, mit der Beschrcibung statistischer Fluktuationen. Diese können besonders im Bereich der Instabilität das Verhalten des Systems bestimmen. $\mathrm{Da}$ auf diese Weise minimale Ursachen weitreichende Folgen haben, führt die synergetische Modcllbildung zu einer modifizierten Kausalanalyse, die der immanenten Dynamik und den Zufallsvariationen mehr Bedeutung beimißt als direkten, linearen Ursache-Wirkungs-Beziehungen.

Die Anwendung dynamischer Konzepte aus der in (1) genannten Tradition ist nicht neu in der Sprachgeographie und in der Geographie im allgemeinen. $\mathrm{Zu}$ erwähnen sind die Diffusionstheorien des 19. Jh.s, die allerdings bisher nicht in einem exakten Theorierahmen formuliert wurden. Ansätze dazu bieten etwa die Arbeiten von Hard (1972) und Kohlheim (1981). In der Soziologie gibt es ebenfalls eine auf Carey (um 1858) und Ravenstein (1885) zurückgehende Tradition, die neuerdings im Rahmen der Agrarsoziologie (vgl. Kiefer 1967) wiederaufgegriffen wurde. Die Gesamttradition dynamischer Theorien im Bereich der Geographie wurde zuletzt in Coffey (1981) systematisch aufgearbeitet, wobei insbesondere die Tradition der Morphologie, die von Goethe über d'Arcy Thompson zu René Thom führt (vgl. Wildgen 1984G), gewürdigt wurde (vgl. Coffey 1981, Kap. 5: Spatial Structure (II) Morphology: Topology). Im Bereich der Regionalund Stadtgeographie liegt bereits eine große Anzahl dynamik-orientierter Arbeiten vor (vgl. die Bibliographie von Zeemann 1981). Zu nennen sind besonders die geographischen Modelle der Brüsseler Forschungsgruppe (etwa Allen 1982) und die Arbeiten von Weidlich (1972) sowie Weidlich/Haag $(1980,1983)$, auf die wir in den folgenden Kapiteln näher eingehen werden. Da das dynamische Paradigma in der Geographie und in der Soziologie (sowie in der Ökologie und der Ökonomie) schon etabliert ist, ist eine Anwendung in der Soziolinguistik eigentlich überfällig. Die speziellen Anwendungen, die wir skizzieren werden, geben aber nur einen ersten Eindruck. Die Soziolinguistik scheint als Ganzes gerade wegen der typischen Verschränkung von interner Dynamik und vielfältiger äuBerer Determination, die nur statistisch zu erfassen ist, ein ideales Feld für die Anwendung synergetischer Methoden zu sein. Im Gegensatz zum Kern der Sprachtheorie (siehe die Archetypensemantik in Wildgen 1982, 1985) bieten die synergetischen Modelle Hakens und die thermodynamischen Ansätze der Brüsseler Schule bessere Möglichkeiten als die eher morphologisch-qualitative Katastrophentheorie. Wir werden jedoch sehen, daß beide Bereiche des dynamischen Paradigmas sich in idealer Weise ergänzen können. 


\section{Sprachwechsel in einer urbanen Sprachyemeinschaft: ein erster Zugang}

Die Sprachveränderung als Wechsel der dominanten Sprache in einem Feld konkurrierender Sprachen öffnet den Blick auf die Makroebene des Sprachwandels. Die Entitäten, welche sich verändern, sind nicht Laute oder Regeln (diese verändern sich außerdem), es sind vielmehr ganze Sprachen, welche im Extremfall entstehen und vergehen, sich in den meisten Fällen aber nur in ihrer gesellschaftlichen Rolle ablösen oder verdrängen. Untersucht man die Bühne, auf der die Sprachen quasi die Handlungsträger sind, im Detail, so kommen Sprachund Sprecherbewertungen sowie Veränderungen in der Sprachbewertung ins Blickfeld. Wir sehen, wie die Bewertungen verschoben werden; sprachliches Prestige wird zugeteilt und abgesprochen, Personen übernehmen oder bekämpfen eine Sprachform, unterwerfen sich ihr, suchen nach Kompromissen usw. Der einzelne Sprecher hat einerseits situative Erfahrungen über den „Marktwert“ einer Sprache, andererseits erlebt er den Bewertungsprozeß häufig passiv als einen überindividuellen Vorgang. Plötzlich glaubt er, sich die Sprache seiner Kindheit nicht mehr leisten zu können, sich ihrer schämen zu müssen. Die soziale Realität von Sprachbewertungen und die praktische Orientierung einzelner Sprecher und ganzer Sprecherschichten an ihnen zeigen die Arbeiten von Labov und anderen sehr deutlich (vgl. Labov 1966).

Wir wollen die Dynamik des Sprachwechsels untersuchen, und zwar anhand des Wechsels der dominanten Umgangssprache in Bremen zwischen 1880 und 1920. Die Wahl dieses Beispiels ist durch eine laufende empirische Arbeit des Autors motiviert, der zu diesem Thema bereits 50 ältere Bremer und Bremerinnen interviewt hat. Unser Modell ist insofern aber provisorisch, als keine exakten und repräsentativen Daten vorliegen, welche eine statistische und quantitative Modellierung ermöglichen würden. Aus diesem Grunde sichert der empirische Hintergrund nur die. Relevanz des Modellbildungsprozesses, ohne jedoch das Ergebnis genau kontrollieren zu können.

\subsection{Zur historischen Dynamik der niederdeutschen Sprache in ihrem Verhältnis zum Hochdeutschen}

Wir wollen zuerst die Makrodynamik der niederdeutschen Sprache bzw. ihrer regionalen Dialekte seit dem 16 . Jh. betrachten, um uns dann spezifischer mit der Jahrhundertwende, besonders der Sprachsituation in Bremen zwischen 1880 und 1920 zu beschäftigen. Möhn (1973) unterscheidet zwei Rezeptionsphasen des Hochdeutschen im traditionellen niederdeutschen Sprachgebiet. Die erste fübrt zur Ablösung des Niederdeutschen als Schriftsprache durch das Hochdeutsche und ist Mitte des 17. Jh.s abgeschlossen. Die zweite betrifft die Verdrängung der niederdeutschen Sprechsprache durch das Hochdeutsche. Sie beginnt im 19. Jh., wird besonders in den großen Städten seit Mitte und gegen Ende des 
19. Jh.s verstïrkt und ist als Gesamtbewegung noch nicht abgeschlossen oder sogar in ihrer 'Tendenz. gebremst.

Abgesehen vom südlichen [llbostfälisch, deren Sprecher bereits im 14. und 15. Jh. zum Mitteldeutschen ühergingen, wobci auch breitere Bevölkerungsschichten den Sprachwechsel vollyogen (vgl. Peters 1973), war die Re/eption des Hochdeutschen im übrigen niederdeutschen Raum \%unächst und für lange Zeit auf dic Schriftsprache beschränkt. Dic Übergangsphase ist zwar insgesamt, bezogen auf das gan\% Gebiet und unterschiedliche soziale Bereiche, über eineinlatlb Jahrhunderte gestreckt; in ein\%elnen Bercichen wurde der Sprachwechsel jedoch schr schnell, etwa in einer Generation vollzogen. In den Kanzlcien sind die meisten Übergänge in den Jahrzehnten von 1540 bis 1560 festzustellen, in einzelnen Regionen und Städten wurde das Niederdeutsche aber erst wesentlich später vcrdrängt (so führte Lübeck scin Oberstadtbuch bis 1809) auf Niederdeutsch, vgl. Sodmann 1973: 119, Fn. 8). Der rasche Wandel ist durch eine gan/e Reihe von Faktoren begünstigt worden. Glohal gesehen, bestand wohl bereits cine länger andauernde Tendenz. der Expansion miltel- und oberdeutscher Sprachforinen, besonders seit dem Sprachausgleich im ostmitteldeutschen Raum gegen Ende des 15. Jh.s Die starken politischen und kulturellen Bewegungen des ausgehenden 15 . und 16. Jh.s verändern die groBräumige politische und ökonomische Konstellation zuungunsten der Hansestädte und ihres Hinterlandes (vgl. Gabriclsson 1980: 120f.). Der Versuch, den Prozeß des Sprachwechsels kausal 7.u erklären, d.h. einige Ursachen für den Wechsel verantwortlich zu machen, stößl auf große Schwierigkeiten. Aus synergetischer Sicht scheint es plausibler, von einem langfristigen Diffusionsgradienten Süd-Nord auszugehen, der durch dic Hanse und ihre überregionale Urkundensprache vorübergehend stark abgeschwächt wurde. Mitteldeutsche Zentren bildeten sich dann im ostdeutschen Siedlungsraum aus (vgl. die Hypothese der Ausgleichssprache von Frings). Mit der Umstrukturierung des Machtgefüges in Norddeutschland (Konflikt der Hanse mit den nordeuropäischen Nationalstáaten) und der erfolgreichen ökonomischen Expansion süddeutscher Städte (Nürnberg, Augsburg) hricht die tragende politisch-ökonomische und kulturelle Basis des Mittelnicderdeutschen zusimmen. An der darauf folgenden raschen Umstrukturierung sind fast alle Berciche des ölfentlichen Lebens (Religion, Schule, Buchdruck, Literatur u.a.) beteiligt. Da dic Veränderung aber nur auf einem hohen soziokulturellen Plateau erfolgt, sind nur dic Führungsschichten und die Schicht der Gebildeten von dem Sprachwechsel direkt betrofien. Man könnte mit einem einfachen Bilde von einer Oberflächenwelle sprechen, welche mit der politischökonomischen Umstrukturierung einhergeht, aber die breite Volkssprache nur unwesentlich berührt. In Abb. 1 stellen wir diesen Gradienten dar, welcher eine Welle bis zum Ausgleich der Sprachunterschicde auslöst.

Allerdings hat das Verschwinden der mittelniederdeutschen Hochsprache, die den niederdeutschen Dialekten als kulturcller Bezugspunkt diente, eine sekundäre Folge. Der Gradient niederdeutscher Dialekt $\rightarrow$ niederdeutsche Standard- 

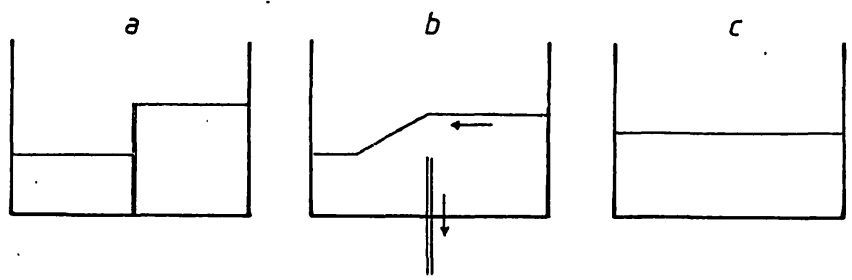

Abb. I

sprache wird durch einen Gradienten: niederdeutscher Dialekt $\rightarrow$ hochdeutsche Standardsprache ersetzt. Dieser neue Gradient ist ursprünglich schwach, da sich das Hochdeutsche vorerst auf den schriftlichen Bereich beschränkt und die breite Masse der Niederdeutsch sprechenden Bevölkerung mit der schriftlichen Sprache nur wenig Kontakt hat. Die erste Folge dieser Umschichtung ist jedoch eine natürliche Diversifikation der Dialekte. Der neue Gradient niederdeutscher Dialekt $\rightarrow$ hochdeutsche Standardsprache verstärkt sich langsam sowohl wegen der zunehmenden Alphabetisierung und der hochdeutschen Einflüsse auf die Sprechsprache der prestigetragenden Schichten als auch durch den Verlust des überdachenden Mittelniederdeutschen. Wir können somit von einem langsamen Zunehmen des Gradienten ausgehen, so da $B$ es ab einem gewissen Punkte zu einer Instabilität der Beziehung zwischen Niederdeutsch und Hochdeutsch kommt. Gegen Ende des 19. Jh.s kommt es in den Bereichen, wo der Gradient und die Auswirkung der äußeren Faktoren am stärksten sind, in den Großstädten zu einer „Ausgleichskatastrophe" in der Richtung des Gradienten Hochdeutsch. Im 19. Jh. sind solche äußeren Faktoren, etwa die Industrialisierung und die damit verbundene Urbanisierung; Nebenwirkungen sind die allgemeine Schulpflicht (in Bremen seit 1844), die Errichtung des deutschen Reiches (AbschluB 1870) und starke Migrationsbewegungen im Zuge der Industrialisierung. Welcher dieser Faktoren exakt welche Folgen hatte, ist kaum feststellbar und im synergetischen Modell auch nicht von zentraler Relevanz, da bei hoher Instabilität eines Systems minimale, an sich bedeutungslose Faktoren den weiteren Verlauf bestimmen können. Insofern führt die dynamische Modellbildung uns von der unlösbaren Aufgabe direkter Kausalanalysen weg zu einer Konstruktion der Entwicklungsgradienten, der Stabilitätsbedingungen und zu einer statistischen Theorie des Wechsels in der Phase der Instabilität. Wir wollen diese Phase etwas genauer am Beispiel Bremens analysieren. 


\subsection{Kontexte des Sprachwechsels in Bremen zwischen 1880 und 1920}

Wegen sciner westlichen Lage war Bremen von der Schwerpunktverlagerung vom Osthandel zum Westhandel weniger betroffen als andere Hansestädte (etwa Lübeck). Die Übernahme der hochdeutschen Schriftsprache erfolgte auch erst ziemlich spät. So wurde das Hochdeutsche in der bremischen Staatskanzlei zwar ab 1530 für die Korrespondenz mit dem Reich, aber erst $1630 \mathrm{im}$ internen Gebrauch cingeführt. Die Beziehungen zu Holland, die auch durch die Übernahme der reformicrten Lehre (ab 1584) gefördert wurden, führten zu einer leichten Konkurrenz zwischen Hochdeutsch und Niederländisch als Sprachen des Handels und der Bildung.

Vor 1870 befand sich Bremen noch in einem vorindustriellen Zustand. Nach dem deutsch-französischen Krieg (1870-1871) begann ein wechselvoller Abschnitt der bremischen Geschichte. Bremen trat zwar spontan dem Reich bei, behielt aber sein eigenes Zollgebiet. Die restriktive Außenhandelspolitik des Reiches brachte Bremen in die verzwickte Lage, daß die Zollfreiheit einerseits die Hafentätigkeit förderte, andererseits aber Industrieansiedlungen außerhalb des Gebietes des Zollvereines wegen der hohen Zollschranken verhinderte. Die unsichere Position gegenüber Preußen und besonders gegenüber Bismarck wurde verschärft durch die notwendige Weserkorrektur, die Bremen allein nicht finanzieren konnte. Nachdem bereits 1879/80 die Eisenbahnlinie OsnabrückBremen-Hamburg verstaatlicht worden war, fand 1888 der Zollanschluß statt; Preußen baute den Bahnhof in Bremen, und mit der Weserkorrektur konnte die Expansion der Dampfschiffahrt beginnen. In den Jahren nach $1890 \mathrm{kam}$ es in Bremen zu einer Wirtschaftsblüte (die Bremer Handelskammer sprach 1901 von ,industrieller Hochflut", cf. Schwarzwälder 1976: 469), die zusammen mit der systematischen Erweiterung des Stadtgebietes und einer rapide steigenden Bevölkerung (1870: mehr als 80000, 1888: 123 500, 1910: 245000) eine tiefgreifende Veränderung der Sozialstruktur nach sich zog. Die vormals ländlichen Randgebiete wurden urbanisiert, die städtische Kultur wurde zu einem Schmelztiegel, in dem alte soziale Beziehungen gelockert und neue aufgebaut wurden. Es findet also eine soziopolitische Umformung statt, welche die ganze Bevölkerung erfaßt. Der soziale Optimismus (vgl. Schwarzwälder 1976: 479, der von einer Zeit spricht, die in ,fast grenzenlosem Optimismus an den wirtschaftlichen und technischen Fortschritt glaubte") und die soziale Neuorganisation verbunden mit regionaler und überregionaler Migration erschütterten auch die bisherige Stabilität der sprachlichen Situation und gaben den Weg frei für eine Umformung in der Linie des seit Jahrhunderten stetig stärker gewordenen Gradienten hin zur hochdeutschen Standardsprache. 


\subsection{Ein qualitatives dynamisches Modell des Sprachwechsels}

Die Bewegung, die diese Jahre von 1880-1920 in die bremische Sprachlandschaft brachten, kann im dynamischen Modell als langsame Dynamik entlang eines Weges in einem bipolaren Feld (niederdeutsche Stadtsprache bzw. ländlicher niederdeutscher Dialekt - vs überregionale hochdeutsche Standardsprache) beschrieben werden. Dem bipolaren Feld entspricht eine Konfiguration von zwei Attraktoren, d.h. von zwei dynamischen Feldern, in denen sich die Bewegung eines Systems stabilisieren kann (dies sind mögliche stabile Zustände des Systems). In Abb. 2 sehen wir eine Folge solcher Attraktorenpaare (Paare von Minima, wobei die Tiefe des Attraktors seine globale Anziehungskraft bzw. seine relative Dominanz) anzeigt. Die beiden stabilen Zustände sind:

$\mathrm{N}$ : Benutzung des Niederdeutschen als Umgangssprache

H: Benutzung des Hochdeutschen als Umgangssprache.

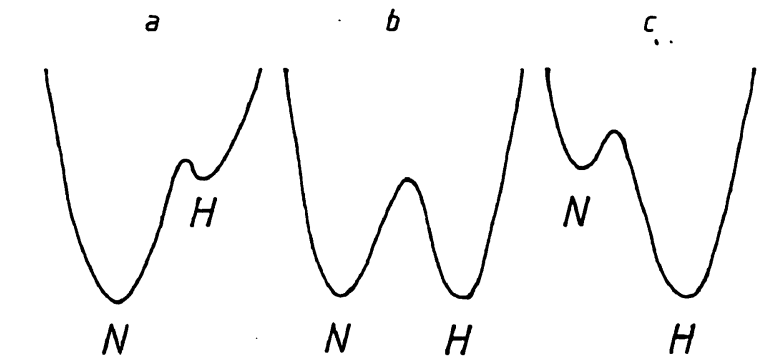

Dominanz des Gleichgewicht Dominanzdes
Niederdeutschen linstabil) Hochdeutschen

Abb. 2

Die Tendenz, die in der Geschichte (s. Abschnitt 2.1) aufgebaut wurde und die mit einem Prestige-, Macht-Gefälle in der Gesellschaft verknüpft war, ist eine weitere Größe; wir können sie als einen Weg im bipolaren Feld ansehen, der langsam von den Bereichen, in denen $\mathrm{N}$ dominiert (s. (a)), zu jenen Bereichen führt, in denen $\mathrm{H}$ dominiert. Zusätzlich wissen wir aus der Geschichte, daß das polare Feld selbst entstanden ist, d.h., daß es auch Situationen ohne Polarität gab (in unserem Falle etwa im 16.Jh. als das Hochdeutsch als Umgangssprache praktisch nicht existierte, jedenfalls in Bremen keine stabile Existenz hatte). Diese qualitativen Beziehungen lassen sich in einem Modell darstellen, das in der Katastrophentheorie „Kuspe“ heißt und das durch die Potentialfunktion (1) definiert ist. 


$$
v=x^{4}+u x^{2}+v x
$$

Was uns interessicrt, ist das Gradientenfeld; dessen Form ist durch die partielle Ablcitung von (1) nach der Variablen $\mathrm{x}$ bestimmt.

(2) $\operatorname{grad} V=\frac{d V}{d x}=4 x^{3}+2 u x+v$

Die Nullstellen der Gleichung (2) geben uns die Attraktoren (Minima) und die Instabilitätspunkte an. Wir erhalten:

(3) $\frac{d V}{d x}=0=4 x^{3}+2 u x+v$

Diese Funktion wird in Abb. 3 als obere Fläche dargestellt.

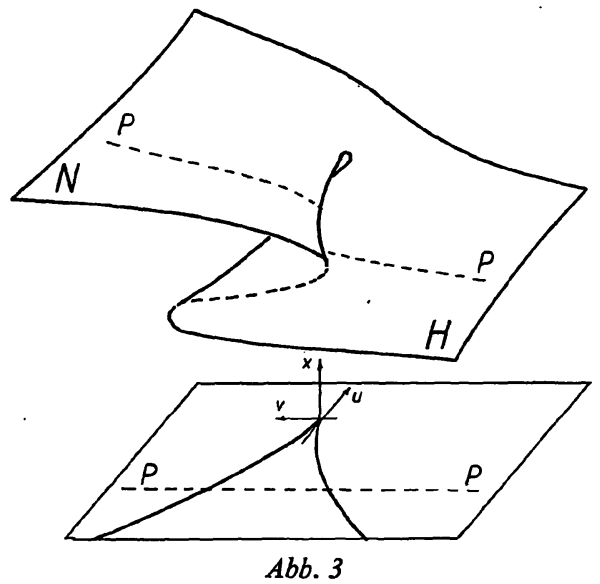

Die Variablen $\mathrm{x}, \mathrm{u}, \mathrm{v} \cdot$ benennen wir als:

$\mathbf{x}=$ Zustandsvariable des Systems; sie gibt die Richtung des Vektorfeldes an,

$v=$ Richtung der langsamen Bewegung,

$\mathrm{u}=$ Richtung, welche die Polarität entstehen bzw. verschwinden läßt (historische Makrodimension).

Wir können Abb. 3 weiter vereinfachen, indem wir nur die Konturen der Faltung, die Bifurkationslinien, betrachten und die Zustandsvariable $x$ vernachlässigen. Dann ist $\mathbf{P}$ eine Linie parallel zur Variablen v. 


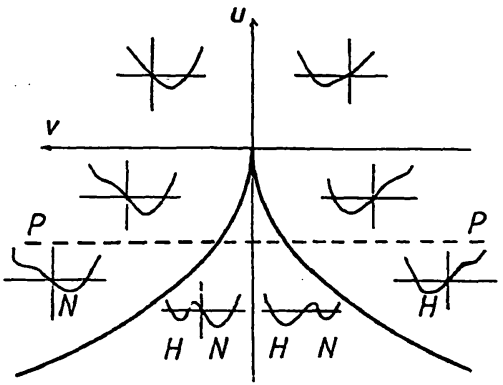

Abb. 4

In der mittelfristigen historischen Entwicklung verändert sich das System entlang des Weges $P$ und gelangt dabei immer näher an jene Linie, an der die beiden Sprachen eine gleiche Anziehungskraft ausüben, oder überschreitet diese Linie gar. Wenn das System extrem ruhig, d.h. ohne Zufallschwankungen wäre, würde es erst den Zustand wechseln, wenn die Sprache N keinerlei Anziehungskraft, Wert, Prestige mehr besitzt. Tatsächlich ist ein System mit vielen Sprechern, das durch die Prozesse des Generationenwechsels und der damit verbundenen Sprachübertragungen, Lernprozesse und durch Migrationseffekte und andere soziale Prozesse „gestört“ wird, nicht ruhig, sondern zufällig fluktuierend. Wir

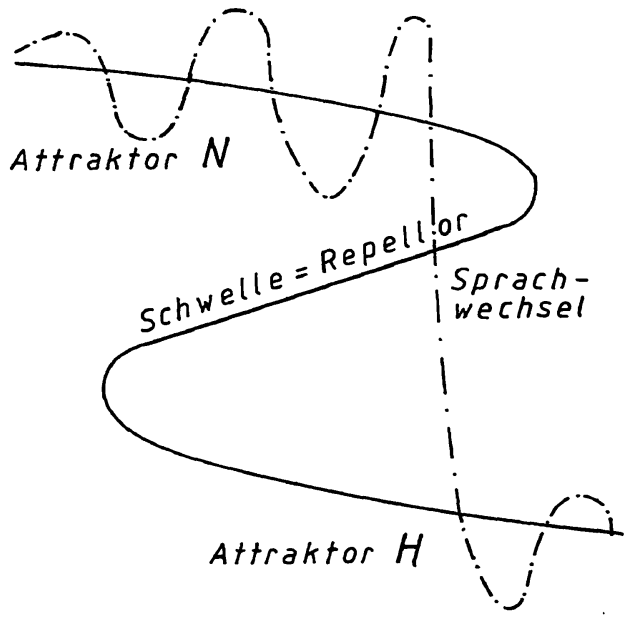

Abb. 5 
stellen diese Fluktuationen ganz provisorisch als Abweichungen vom Attraktor dar und benutzen dazu einen Schnitt durch Abb. 3, so daß der Weg P in diesem Schnitt verläuft. Die Fluktuation führt das System über die Zwischenlinien mit Repelloren (Maxima) hinaus und bringt somit das System in das Anziehungsfeld des Attraktors $\mathrm{H}$. In unserem Modell gibt es also zwei klar unterscheidbare Bedingungen für den Sprachwechsel:

(a) Der historische (langsame) Proze $B$ entlang des Weges $P$ ist in einem Bereich angelangt, wo dic Anziehungskraft des Attraktors $\mathrm{H}$ ausreichend stark ist. Dieser langsame Prozeß verläuft ziemlich kontinuierlich; allerdings zeigt er keine starken Wirkungen, solange die Situation global stabil ist.

(b) Die Fluktuation im Feld muß ausreichend stark sein. Ihr Effekt kann additiv durch die Auflösung lokaler Ordnungen (etwa durch die Urbanisierung), durch starke innere oder äußere Migration und durch die Erschütterung des bisherigen Wertgefüges, durch die Etablierung neuer sozialer Zielvorstellungen (sozialer Aufstieg), die Zerstörung tradierter Rollenmuster (etwa bei der Berufstätigkeit der Mutter) usw. ausgelöst werden. Außerdem können sich all diese Determinanten kooperativ verstärken. Wesentlich ist jedoch, daß die dynamische Konfiguration einerseits und die Fluktuation andererseits in spezifischer Weise zusammentreffen. In dieser Situation gibt es dann einen Erdrutsch, d. h., die Bewegung, die in unserem Beispiel seit dem 17. Jh. angelegt war, entlädt sich in einem Prozeß des Sprachwechsels. Diese Bewegung kann sich direkt im Sprachverhalten (situative Anpassung an die andere Sprache) oder retardiert durch die Kindererziehung auswirken oder kombiniert in beiden Bereichen.

Um diese Vorhersage des Systems plausibler zu machen, will ich einige Fakten anführen, die den genannten Erdrutscheffekt bestätigen.

\subsection{Einige empirische Sachverhalte zum Sprachwechsel in Bremen}

\subsubsection{Befragungsergebnisse}

(a) In den Jahren 1938-1939 wurden im Rahmen der Erhebung des „Niederdeutschen Wörterbuches" auch in Bremen die Eltern von Schulkindern an einigen Schulen schriftlich befragt. Wir wollen die Ergebnisse tabellarisch zusammenfassen (vgl. Janßen 1943: 19, sowie 65-68). Es sprechen Plattdeutsch (mit Unentschiedenen): 


\begin{tabular}{l|c|c|c} 
& $\begin{array}{c}\text { Eltern } \\
\text { untereinander }\end{array}$ & $\begin{array}{c}\text { Eltern mit } \\
\text { ihren Kindern }\end{array}$ & $\begin{array}{c}\text { Kinder mit } \\
\text { Schulkameraden }\end{array}$ \\
\hline $\begin{array}{l}\text { Bremer } \\
\text { Stadtgebiet }\end{array}$ & $26-29 \%$ & $11-26 \%$ & $0-11 \%$ \\
\hline $\begin{array}{l}\text { Bremer } \\
\text { Außenbezirke } \\
\text { (Borgfeld, }\end{array}$ & & & \\
$\begin{array}{l}\text { Osterholz, } \\
\text { Rablinghausen) }\end{array}$ & $65-75 \%$ & $29-43 \%$ & $34-45 \%$
\end{tabular}

Diese Statistik zeigt deutlich das Zentrum-Peripherie-Gefälle und die dramatische Abnahme von der Elterngeneration (im Durchschnitt um 1900 geboren) zur Kindergeneration (im Durchschnitt um 1930 geboren).

(b) Eine weitere Befragung, die ebenfalls schriftlich über die Schulen erfolgte, hat 1965 Traute Brüggebors durchgeführt (2265 ausgewertete Fragebögen). Anhand ihrer Ergebnisse muß die Hypothese eines einfachen Gefälles Stadtzentrum-Peripherie relativiert werden. Durch Neuansiedlungen an der Peripherie ist dort der Anteil Zugewanderter Nicht-Platt-Sprecher größer, d.h., aus einer ländlichen Peripherie um die Jahrhundertwende ist teilweise eine StadtfluchtPeripherie mit entgegengesetzter Sprachwahltendenz geworden.

(c) Ein neueres Ergebnis auf der Basis von 1033 durchgeführten Interviews erbrachte die vom Niederdeutschen Institut in Auftrag gegebene GETAS-Studie. Eine der Fragen lautete:

Können Sie Plattdeutsch sprechen.

Ja, gut

$\mathrm{Ja}$, ein bißchen

Nein, gar nicht.

Die Antworten zeigen eine deutliche Abhängigkeit der Ergebnisse von den Faktoren: Alter und Ansässigkeit. Wir verwenden nur die Teilantwort ,,ja, gut" für unseren Vergleich.

\begin{tabular}{l|c|c|c|c} 
Alter & $18-29$ & $30-44$ & $45-59$ & $60-$ \\
\hline ja, gut & $6 \%$ & $19 \%$ & $32 \%$ & $47 \%$
\end{tabular}

!Die deutliche Altersabhängigkeit der Sprachbeherrschung (immerhin findet eine Reduktion auf fast $1 / 8$ statt) weist auf eine starke und schnelle historische Veränderung hin. Ergänzt wird dieses Bild durch die Verteilung nach Ortsansässigkeit. 
Wohnhaft in Bremen

\begin{tabular}{c|c|c}
$\begin{array}{c}\text { von } \\
\text { Gcburt an }\end{array}$ & $\begin{array}{c}21 \\
\text { mehr Jahre }\end{array}$ & bis 20 Jahre \\
\hline $35 \%$ & $29 \%$ & $15 \%$
\end{tabular}

Die Öffnung der niederdeutschen Sprachgemeinschaft für Zugewanderte ist offensichtlich gering; praktisch ist es für Personen, die heute nach Bremen ziehen und keine verwandtschaftlichen Beziehungen hier haben, unmöglich, im natürlichen Umgang den Ortsdialekt zu lernen (selbst wenn sie es wünschten).

\subsubsection{Angaben in Lebenserinnerungen und autobiographischen} Romanen zur Umgangssprache in Bremen zwischen 1870 und 1900

(a) Georg Droste beschreibt in seinem Roman „Ottjen Alldag“ die Kindheit eines plattdeutschen Jungen in Bremen nach 1870. Ottjen wächst in einer Kate am Osterdeich auf und erlebt noch in der Kindheit, wie neue Häuser am Deich die alten verdrängen und wie damit auch die hochdeutsche Umgangssprache in sein Leben tritt. Von den Hochdeutschen am Deich, ,dat ol' dickkoppde Volk an'n Diek" berichtet Frau Rosenbohms, daß ihre Dienstboten, kaum der. Bauerndorf entronnen, sich für das Plattdeutsche schon zu fein waren (Droste: 1913, Bd.1: 160):

„Kam't von'n Burndorpen un dreiht denn dat ole Snutwark, just as wenn se: nu up'n mal keen Platt mehr könt! ,Heute for Behn Fennige mehr, Frau Rosen. baums!' geiht'dat denn, un denn scholl'n jem doch den ganzen Stutenkorf an dal. ole Polkapriek flenzen! Is dat upstunns noch'n Welt?"“.

Hochdeutsch ist zwar Ottjens Zweitsprache, aber seine Mutter sieht dock. seinen Umgang mit den hochdeutschen Nachbarnkindern aus den neuen Häusern am Deich mit Wohlwollen. Aus Ottjen soll etwas Besseres werden, er soll es besser haben. Der Vater duldet die Ambitionen der Mutter.

Als Ottjen mit gutem Zeugnis (gefördert durch den hochdeutschen Nachbarn Dr. Görtz) beim Tabakshändler vorspricht wegen einer Lehrstelle, ist ihm sein Platt immerhin nützlich: „Darr harr he mit dit Tügniß, un mit'n Breef, den de Dokter em upschräben harr, nah d'r Langenstraten henngahn moßt, nah so'n grotet olet Tobacksgeschäft. De frundlige Inhebber von dit Geschäft harr sick'n ganze Tied wat mit em vertellt, un harr sogar 'n feinet gemütliget Platt mit em snackt.“(Bd.1:207). Als Ottjen dann seine Lehrzeit beginnt, muß er erst lernen, mit wem er platt und mit wem er hochdeutsch zu sprechen hat. Im Packhaus war Platt geboten: „Hier moß he so snacken, un sick so geben, as he achtern Diek baren und tagen weer: Platt un deftig." (Bd. 2: 11). Im Kantor ist Hochdeutsch geboten, obwohl die Alteingesessenen und fest Etablierten frei variieren können, so etwa der alte Kaufmann oder die „Tobbacksnäse“ Adam (Bd.2: 17). 
Insgesamt gibt der Roman, der breiteste Anerkennung in Bremen fand, das Sprachklima der Jahrhundertwende aus der Sicht der um sozialen Aufstieg be; mühten Familie im Stadtkern sehr gut wieder. In den Außenvierteln fand dersel: be Umschichtungsprozeß 10-20 Jahre später statt.

(b) In dem autobiographischen Roman „Der Heini von Bremen“ von Heinrich Binder (1923) beschreibt der Autor, wie seine Mutter sich als Fremde in Bremen zurechtfinden mußte. Die Szene muß sich etwa 1890 abgespielt haben: , Es kam noch hinzu, daß das Einleben in die fremde Stadt für Jupp und die ganze Familie Kröger genug der Sorgen und Gedanken brachte. Vor allem sah Frau Kröger ein, daß sie ernste Sprachstudien treiben müsse. Sie sprach eine Mischung von Hochdeutsch, Englisch und Rheinpfälzisch, wohingegen die Markt- und Fischfrauen der Freien und Hansestadt Bremen ein Plattdeutsch ? von unverwässerter Reinheit und Feinheit sprachen" (Binder 1923: 29).

(c) In dem Bericht von Gustav Röhrs: „Bremen-Ost. Erlebtes und Erlauschtes $\because \mathrm{i}_{\mathrm{i}}$ eines Bremer Jungen“ wird der Konflikt zwischen den Großeltern, die noch Platt $\therefore$ sprachen, und der nächsten Generation deutlich gemacht. Allerdings spielt diese Geschichte in einer wohlhabenderen Schicht; wodurch ein relativ früher Sprach$\because\lrcorner$ wechsel erklärt wird. Die Großmutter spricht mit ihrer Schwiegermutter noch $\therefore$ :i: Platt: „Och, - n'obend Tine, häs mol'n Ogenblick Tied forr mi, mien Deern?“ 1:. , ,Ober gewiß doch Muddern, solang du willt, - kumm, sett di henn.“ (Röhrs 1976: 21). Mit den Enkeln aber darf nur Hochdeutsch gesprochen werden, wie i- $\quad$ die folgende Episode lebendig illustriert: „Oma guckte aus dem Fenster, - rich.. tig, die Kinder ihrer Schwiegertochter Tine standen vor der Tür. Fünf an der ..... Zahl. Ja heute war ihr Besuchstag. Oma rief Didi zu: ,Heer mien Jung', fang den Schlödel upp', was auch prompt geschah ... Richtig, es fiel ihr noch zur rechten te Zeit ein, Hochdeutsch möchte sie mit den Kindern sprechen, so wünschte es ihre : Schwiegertochter: , $\mathrm{Na}$ - Kinder, wollt' ihr Eure alte Oma mal wieder besuchen? : Heut ist ja Euer Besuchstag.“” (Röhrs 1976: 19).

An dieser Episode wird deutlich, daß der Sprachwechsel sich eben nicht über $y$ 'Prozesse der stetigen Übernahme von neuem Sprachgut vollzog, daß vielmehr ind systematisch an der Bruchstelle der Generationen die Weitergabe der tradierten $\because \in:$ Sprache blockiert wurde. Nur durch diesen Eingriff an der empfindlichsten $\because$ Nahtstelle der Sprachtradierung ist der abrupte Wechsel überhaupt erklärbar. : Das bedeutet aber, daß der Prozeß, der die ganze Sprachgemeinschaft erfaßte, - dem Einzelnen, in diesem Fall den Kindern, den Sprachwechsel aufgezwungen - hat. Allerdings sind die Träger des Zwanges, etwa die um den sozialen Aufstieg ihrer Kinder besorgte Mutter, selbst nicht frei; sie unterwerfen sich einer kollek3 tiven Dynamik. Der Sprachwechsel enthält somit zwei Phasen, von denen die ! erste globale kollektive Zwänge erzeugt, die zweite einen lokalen, familieninterinen Zwang. 
Phase A: Die Elterngeneration hält plötzlich die alte Sprache für nicht mehr tragbar und optiert projcktiv (auf die Chance ihrer Kinder bezogen) für dic neue Sprache.

Phase B: Die Kinder werden durch familieninterne Regelungen der Kommunikation auf die neue Sprache hin ausgerichtet.

(d) Ein anderer autobiographischer Bericht von Anton Kippenberg (,Geschichten aus einer alten Hansestadt") schildert persönliche Erlebnisse aus Bremen ab ca. 1880. Dabei fallt eine typische Ambivalenz auf. Der Autor, der aus besserem Hause ist, hat „zu Hause und im täglichen Umgang nur hochdeutsch" gesprochen (Kippenberg 1942: 55f.) und berichtet, daß die Kinder der armen Bevölkerung etwa aus dem Stephaniviertel noch Plattdeutsch gesprochen hätten. Obwohl diese soziale Verteilung der Sprachen für den Autor feststeht, werden sprachkonservative Einzelindividuen der sozialen Oberschicht als Vorbilder hervorgehoben.

„Richter Smidt pflegte nicht nur im Gerichtssaal, sondern auch zu Hause das urgewachsene und urwüchsige Platt zu sprechen, das trotzig die zweite Lautverschiebung so wenig mitgemacht hatte wie die Friesen die Völkerwanderung. Auch Dr. Thulesius, den wir bald kennenlernen werden, sprach nur Platt. Wie denn überhaupt gerade manche der alten und bevorzugten Familien der Hansestadt bis weit in die Hälfte des neunzehnten Jahrhunderts hinein zäh an der alten heimatlichen Sprache festhielten". (Kippenberg 1942: 136).

Hier stehen die „zweite Lautverschiebung“" pseudowissenschaftlich für die wirtschaftlichen und kulturellen Einflüsse aus dem Süden, die Friesen müssen als Symbol des trotzigen Beharrens auf dem eigenen Raum und der eigener Kultur herhalten. Beides bejahend kommt der Bremer Autor und mit ihm viele sozial Arrivierte in ein Dilemma, das sie dadurch lösen, daß sie dem Plattdeut.schen eine eigene Kulturrolle neben dem herrschenden Hochdeutschen zuwei-. sen. Diese Ambivalenz kommt auch in vielen Sprachbiographien meines Korpus zum Vorschein.

\subsubsection{Inhaltliche Analyse der Angaben in sprachbiographischen Interviews}

Um Einzelheiten zu diesen Prozessen auf dem Gebiet individueller Einstellungen und Verhaltenssteuerungen zu gewinnen, habe ich bis dato 50 alte Bremer interviewt, wobei die Methoden der „Oral History" und der soziologischen Biographieforschung verwendet wurden (vgl. Niethammer 1980; Matthes/Pfeifenberger/Stosberg 1981). Einzelne Ergebnisse sollen hier herangezogen werden; die Gesamtauswertung erfolgt, sobald das Datenmaterial vollständig ist (mindestens 100 Interviews ausgeglichen nach Geschlecht, Alter, sozialer Schicht und Stadtviertel). 
Für dịe Zeit zwischen 1890-1920 (der älteste Befragte war 1887 geboren) ergibt sich das folgende Bild:

\section{A: Oberschicht}

Sprecherin 8c (geb. 1907)

„... ich bin Bremerin, meine Eltern waren Bremer, meine Großeltern und meine Urgroßeltern, hat kein Mensch Plattdeutsch gesprochen, ich habe hier im Sommer erst auf diesem Landgut bei meinen Großeltern oft gewohnt und da hab ich mit der Tochter vom Hofmeister gespielt... und die sprach Plattdeutsch."

Die Sprecherin ging ins Lyzeum ,und da hat nie jemand Platt gesprochen“. Trägt man diese Angaben in eine Zeitskala ein, so ergibt sich, daß in den besseren und wohlhabenden Kreisen in Bremen bereits Mitte des 19. Jh.s Hochdeutsch gesprochen wurde. Allerdings verfügte man passiv über das Niederdeutsche, da es sowohl auf dem Lande als auch in den unteren Schichten noch eine einsprachige, Niederdeutsch sprechende Bevölkerung gab.

Sprecher $5 b$ (geb. 1891)

Auch in diesem Fall wird die soziale Abwertung des Platt aus der Sicht des Oberschichtzugehörigen deutlich.

Sprecher: So, also nun wollen Sie wissen, was wir da gesprochen haben? Interviewer: Ja!!

Sp.: Deutsch. Richtig Deutsch. Nix Plattdeutsch

Int: Kein Plattdeutsch?

Sp.: Nein. Plattdeutsch wurde nur noch ... in Familien gesprochen.

Int.: Aha!

Sp.: Und, vor allen Dingen, bei der ... will das mal ... etwas sonderbar ausdrücken, bei der einfachen Bevölkerung, d.h. also ... nicht, wo die Kinder der höheren Schulen besuchen, sondern wo sie Volksschule gingen, nich, verstehen Sie?

Trotz dieser sozialen Abwertung hat die Kenntnis des Platts positive Werte; es gehört dazu, Platt zu verstehen. Aber diese Fähigkeit liegt auf einer ganz anderen Ebene als die Beherrschung des Hochdeutschen.

Sp.: Also ich muß ihnen ganz offen sagen ... Meine Frau kann noch Platt snaken, ik kann ok noch Platt snaken. Meine Mutter konnte es ... Es war aber keine offizielle Sprache.

Auf die Frage, wo er denn seine Plattkenntnisse her habe, wenn er praktisch nie die Sprache benutzt habe, antwortet der Sprecher: 
Sp.: Gclernt habc ich das nicht ... Das frißt man so ... normalerweise mit.

Die Erinnerungen an die Benutzung des Platts in der Jugend werden verdrängt.

Sp.: Wir Kinder redeten kein Platt ... Was fürn Kauderwelsch wir gesprochen haben, kann ich ihnen nicht sagen.

Auf Nachfragen zu seiner Sprachpraxis als späterer Kaufmann reagiert der Sprecher entrüstet:

Sp.: (empört) Lieber Mann entschuldigen Sie mal ... Wie kommt Ihr denn auf eine solche Idee, daß ich heute noch wissen soll, wie ich im Hafen geklönt habe? Dat weet ik nich, entschuldigt wol.

Der Kodewechsel im letzten Satz ist verräterisch.

Sprecher 4 (geb. 1887)

Auf die Frage, ob in Kneipen oder Geschäften noch Plattdeutsch gesprochen wurde, antwortet der Sprecher:

Sp.: Wenn die Bremer ihre Köppe zusammenstecken, kann ich mir sehr gut denken, und ich mein das auch noch in Erinnerung zu haben, daß die das auch noch alles plattdütsch verhackstückelten ... Was so ein richtiger Bremer ist, der kann Platt, der muß Platt können.

Die niederdeutsche Sprache ist also in diesen Kreisen noch schwach an die Ortssolidarität gebunden, die passive Beherrschung wird akzeptiert, das aktive und besonders das ernsthafte Verwenden der niederdeutschen Sprache ist deutlich diskriminiert. Beziehungen zum Anpassungsdruck, der von der Schule ausging, sind ebenfalls deutlich. Plattdeutsch wird mit dörflicher Kultur und Volksschule (Freischule) und häufig mit sozialem Mißerfolg (Sitzenbleiben) assoziiert.

\section{B. Mittelschicht (obere, mittlere)}

In der Mittelschicht (Beamte, höhere Angestellte, Handwerksmeister) finden wir die Assoziation Schulversagen und Plattdeutsch ebenfalls, allerdings ist z.T. die Solidarität mit der regionalen Sprache stärker ausgeprägt, wobei man als argumentative Stütze auf einige Patrizierfamilien verweist, in denen ein feines Platt gepflegt wurde.

Sprecherin 3 (geb. 1899)

Int.: Gab es Kinder, die ohne Hochdeutschkenntnisse zur Schule kamen? 
Sp.: Ja die hattens schwer.

Int.: Ja.

Sp.: Das weiß ich, in meiner Klasse waren paar die es sehr schwer hatten. Die kamen ohne Hochdeutsch in die Schule, weil die Eltern nur so sprachen, und das waren natürlich immer Sitzenbleiber und keine besonders begabten Schüler.

Sp.: Das hing also mehr von dem Beruf oder den Bemühungen der Eltern ab.

Die Sprecherin erzählt, daß sie und ihr Mann begeistert plattdeutsche Literatur lasen und daß für sie das Plattdeutsche zum Symbol von Heimatverbundenheit wurde.

Wir finden hier eine mehrfach in den Interviews festgestellte Polarität zwischen der Abwendung vom Platt als Gebrauchssprache unter dem sozialen Druck von Schule, gesellschaftlichem Umfeld und Eltern und der Hinwendung zum Platt als Symbol der örtlichen Kultur. Das Niederdeutsche wird, wie Möhn (1980) dies auch beschreibt, einerseits aus der öffentlich, alltagssprachlichen Kommunikation verdrängt und ,privatisiert", andererseits erscheint es wieder in medienvermittelter Form (im Theater, in Treffen des Plattdeutschen Vereines, später im Rundfunk und Fernșehen).

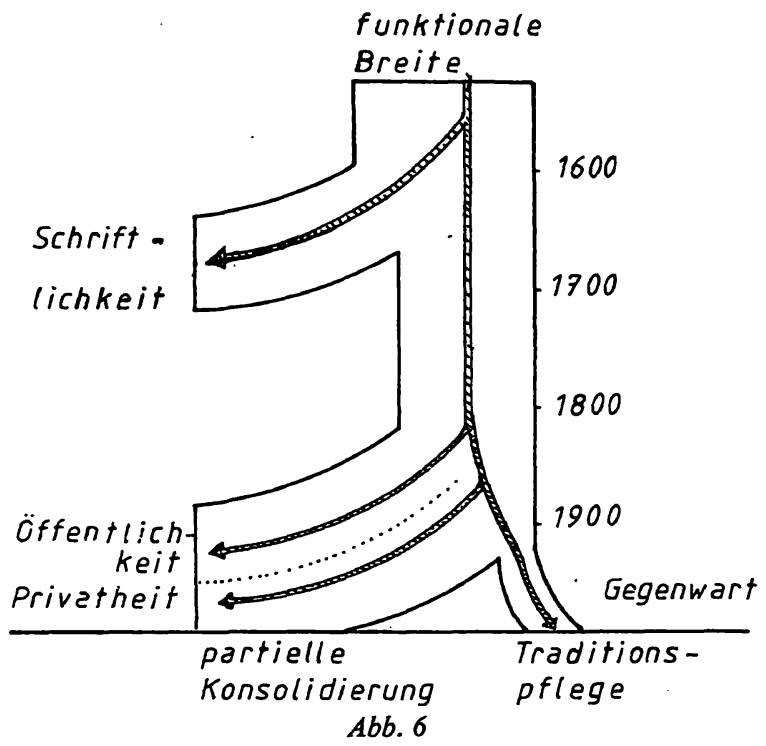


kömnen, König; Sohn, Sonne, sonst, gesponnen, gewonnen, geronnen; Sommer, fromm dürfte so ziemlich der Großteil der Fälle erfaßt sein. Dagegen stehen:

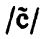

ver/gewöhnen

schön

etc. $|\tilde{\mathrm{o}}|$

schon

wohnen

etc.

die diesen Regeln nicht unterliegen.

Darüber hinaus ist die Argumentation von Felix/Kühl widersprüchlich (besonders ab p. 192, Z. 4 v.u. bezüglich 192, Z. $6 \mathrm{ff}$.): Wenn mittlere Vokalhebung nur auf gerundeten Vordervokalen operiert und Umlaut-Auflösung deshalb nachgeordnet zu sein hat, dann ist natürlich die von Felix/Kühl vorgeschlagene Hierarchisierung (2) > (3) genau umzukehren: Mittlere Vokalhebung geht vor Umlaut-Auflösung. Dies wiederum würde aber die Akzeptabilität von Formen wie $\left[{ }^{*} \mathrm{kyneg} /{ }^{*} \mathrm{kyn} ə n\right]$ voraussagen, was natürlich nicht zutrifft. Dieser - von ihnen übrigens nicht bemerkte - Widerspruch stellt für den Felix/Kühl-Ansatz ein unlösbares Problem dar.

ii) Was hier offenbar auftritt, ist eine dreistufige Ersetzungsmöglichkeit: Zwischen stark dialektalem [i] ([kinẹ, kine $]$ ) und hochsprachlichem $[\phi, \propto]$ (k $\phi$ nik, kœnən) existiert eine ,gemäßigt dialektale“ Variante mit ungerundeter Entsprechung zu hochsprachlichem $[\phi, \propto]$. Für diese Einzelfälle Regeln zu konstruieren (die naturgemäß mehr Ausnahmen aufweisen als Regelfälle) mutet nicht besonders plausibel an - noch dazu, wo im gleichen Atemzug so ,idiosynkratische Formen " wie [blëeme] 'Blumen' ausgeschlossen werden (183), obwohl die mindestens die gleiche Regelmäßigkeit aufweisen die wie besprochenen Fälle, was aus jeder einschlägigen Dialektgrammatik zweifelsfrei hervorgeht.

Mit dieser Auswahl aus dem ,deskriptiven“ Teil lasse ich es hier bewenden; ich denke, daß die Hauptstoßrichtung meiner Kritik im Prinzip bereits deutlich geworden sein müßte. Felix/Kühl diskutieren noch die Interaktion von ,Tiefer

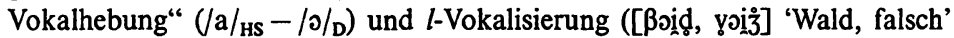
etc.) sowie eine lautliche Besonderheit des Dialekts aus dem Bereich der $r$-Vokalisierung.

\section{Die Hierarchie phonologischer Regeln und ihre „Erklärung“}

Alle diese Regeln werden nun in einem nächsten Schritt zusammengenommen und damit eine „Hierarchie phonologischer Regeln bei Dialektsprechern“ erstellt. Diese sieht folgendermaßen aus ${ }^{3}$ (195):

$3 r$-Vokalisierung und eine weitere Regel habe ich der besseren Verständlichkeit wegen eliminiert. 
Sprecherin $7 a$ (geb. 1902)

Spr.: Nur meine Eltern, die hatten Angst, die ... sprachen immer nur Platt und die Oma auch, die war in Falkenberg geboren, auch bei Lilienthal, nech, aber immer Platt, aber wenn wir Kinder da waren, denn wurde Hochdeutsch gesprochen. De mot richtich Dütsch lern, ... hieß das da, Plattdütsche komt se nich mit klor.

Int.: Und zu Hause so normal

Spr.: ... aber wenn wir Kinder anfingen auch Platt zu sprechen, dann haben meine Eltern immer gebremst.

Die Sprecherin ging in die „Geldschule“, ihre älteren Brüder, die in eine andere Schule gingen bzw. die Schule schon verlassen hatten, durften es sich erlauben, Platt zu sprechen.

Spr.: Und meine Schule, die war nun'n bißchen was Besseres:

Int.: $\mathrm{Hm}$

Spr.: Ja, und da mußte ich da ja nun auch, mußte ja nun auch Hochdeutsch sprechen, nech.

Es gab somit formelle Erziehungssituationen, in denen das Hochdeutsche von den Eltern erzwungen wurde, die selbst Platt sprachen. Außerhalb dieser Situationen wurde vielfach auch von den Kindern Platt gesprochen. Die sozial differenzierte Schule (Freischule-Geldschule) erzwang in Kooperation mit den aufstiegsgläubigen Eltern den Sprachwechsel der Kinder. Daneben gab es ebenso Situationen, in denen Platt erzwungen wurde, so etwa im Plattdeutschen Verein.

Sp.: Ach ja, da war auch n' Vorsitzender, und denn do da wurde nur Platt gesprochen, und wenn man die traf, dann mußte man auch nur Platt antworten.

Diese zwiespältige Situation förderte auch die Sprachmischung, die Sprecherin 7a nennt die Sprache der Kinder deshalb ,halb Platt und halb Hochdeutsch". Innerhalb der Familie gab es die eigenartigsten Differenzierungen, so erzählt Sprecherin $7 b$ (geb. 1908):

Spr.: großen Brüder, ja die sprachen dann Platt. Das ist bei uns so Sitte. Die Großen sprachen Platt und die Lütschen ...

In anderen Familien sprach der Vater mit den Kindern Hochdeutsch, die Mutter aber Plattdeutsch. 
Sprecherin 10a (geb. 1908)

Spr.: Mcin Vater war Buchdruckermeister, aber der machte das so schon, der sprach mit uns nicht viel Plattdeutsch aber Mutter die sprach immer mit uns Plattdeutsch ...

Von ihrer Schwester, die 10 Jahre jünger war, sagt sie:

Spr.: Die hatte das so nicht mehr mitbekommen, daß zu Hause so viel Plattdeutsch gesprochen wurde.

Sprecherin 10d (geb. 1908) berichtet, die Großmutter habe mit ihren Enkeln Platt gesprochen, zu der dazwischen liegenden Generation aber Hochdeutsch.

Spr.: Meine Eltern sprachen aber kein Plattdeutsch, meine Großmutter war bei uns zu Hause, die ist 58 geboren und die sprach nur Plattdeutsch mit mir nech mit meiner Mutter und nicht mein Vater (...) nur mit den Enkeln Plattdeutsch.

Auf diesem Weg hat dann auch der Urenkel Plattdeutsch gelernt, obwohl dessen Großeltern bereits für das Hochdeutsche optiert hatten.

Ganz allgemein war ein Sprachwechsel derart, daß die Eltern untereinander Platt sprachen und dann, wenn sie sich an die Kinder wandten, Hochdeutsch sprachen.

Sprecherin $10 b$ (geb. 1910) berichtet:

Spr.: Die Eltern tosamen die snackt immer bloß platt, nun we konnt da aber ja dann alles verstan, nech, wat se segen, aber wenn we direkt angesproken warn, denn sagten sie das hochdeutsch.

Erwachsene, die generell zum Hochdeutschen übergingen, wurden teilweise als lächerlich angesehen. Dieselbe Sprecherin berichtet von Nachbarn, die vom Dorf stammten.

Spr.: Aber das war nicht mehr vornehm, wenn man Plattdeutsch sprach, die mußten dann Hochdeutsch sprechen und dann hebt we da als Kinder immer drüber lacht.

Einige Sprecher wehrten sich auch gegen die schnelle Sprachanpassung. Sprecherin 10c (geb. 1913) berichtet von ihrer Schwester, die 10 Jahre älter war.

Spr.: Aber da fühlte sie sich zu fein zu, irgendwas war da, was, das wollte sie schon, als ich och noch zur Schule ging (...), da wollte sie in der Straße 
schon immer mit mir Hochdeutsch sprechen, weil sich das besser anhörte, sagte sie, ((flüsternd)) wir sprechen aber unterwegs Hochdeutsch, und das konnte ich nicht, weil mir das einfach komisch vorkam nech.

Sprecherin $11 \mathrm{~g}$ berichtet von ihrer Mutter (geb. 1887)

Spr.: (...) weil mein Großvater ja auch Platt sprach, und mit seiner Familie hat er auch Platt gesprochen, und meine Mutter war die jüngste Tochter, na so jung war sie auch nicht, 87 geboren, und da wollten sie mit ihr Hochdeutsch sprechen, weil das nicht mehr modern war.

Int.: Modern war? ((die Spr. hatte wohl gemeint, daß das Plattdeutsche nicht modern war))

Spr.: Nicht mehr modern war und dann hat sie immer Platt geantwortet und da kam sie mit ihrem Hochdeutsch nicht durch.

In der ländlichen, peripher wohnenden Unterschicht war das Plattdeutsche im ersten Jahrzehnt noch recht stabil, es gab sogar eine bewußte Opposition gegen die Stadt und ihr Hochdeutsch, allerdings gingen die großen Bauern bereits in dieser Zeit dazu über, mit ihren Kindern Hochdeutsch zu sprechen und sich damit sprachlich von den Kleinbauern und Tagelöhnern abzuheben. Generell ergibt sich das Bild einer durch starke Wertveränderungen und Strukturumbildungen destabilisierten Gesellschaft.

$\mathrm{DaB}$ selbst Familien, die Solidaritätsgefühle für das Platt hatten, schließlich mit ihren Kindern Hochdeutsch sprachen, macht die folgende Episode deutlich, mit der ich die kurze Darlegung der empirischen Befunde schließen möchte.

Sprecherin $11 b$ (geb. 1916)

Spr.: (...) mit uns Jüngeren hat Vater auch nicht mehr Mutter auch nicht mehr Plattdeutsch gesprochen, sie sagten plötzlich, da war eine Schranke, da konnten sie nicht mehr und da haben sie mit uns Hochdeutsch gesprochen mit den Jüngeren, wir haben aber gerne Plattdeutsch gehört und habens auch gelernt dabei.

Wenn hier die Sprecherin von „Schranke“ spricht, dann haben wir ganz anschaulich die subjektive Empfindung jenes überindividuellen Prozesses des Sprachwechsels, der wohl aus vielfältigen Quellen Unterstützung erhielt, von vielen aber eher erduldet als herbeigeführt wurde. Die geschilderte Familiensituation zeigt außerdem, daß der globalen gesellschaftlichen Dynamik, wie sie in den Abb. 3 bis 5 schematisiert wurde, eine ähnliche Dynamik in den einzelnen Familien, ja beim einzelnen Sprecher entspricht. So ist einerseits die globale 
Dynamik der Effekt cinzelner Umschwünge, andererseits erhält die globale Dynamik soviel Gewicht, daß sie die Individuen synergetisch gesprochen ,versklavt"• und damit den Gesamteffekt nochmals verstärkt.

Aus den cinzelnen Erinnerungen der damaligen Teilnchmer am Prozeß läßt sich nicht nur das in vielen Dimensionen fluktuierende Geschehen ablesen, es wird auch deutlich, wie wenig durch eine Kausalanalyse gewonnen ist. Nur eine Analyse kooperativer Effekte und partieller Rückkoppelungen im Rahmen eincs synergetischen Modellkonzeptes verspricht, das Geschehen zumindest in wichtigen Aspekten zu erfassen.

Wir wollen im folgenden Kapitel versuchen, anhand eines mathematischen Modells das kollektive Verhalten einer Sprachgemeinschaft unter ähnlichen Bedingungen zu simulieren.

\section{Synergetische Simulation des Sprachwechsels}

Bis jetzt wurde der Prozeß rein qualitativ, d.h. grob, ohne Berücksichtigung der Vielfalt einzelner Bewegungen, individueller Variationen, Bewertungen usw. abgebildet. Wenn wir uns auf die entscheidende Phase der Destabilisierung konzentrieren und dabei versuchen, Beweggründe, Randbedingungen, auslösende Faktoren aufzuklären, wird unser qualitatives Instrumentarium inadäquat. Diese Inadäquatheit ist eine generelle Erscheinung, denn Fluktuationen, welche in stabilen Zuständen gedämpft werden (dies ist quasi die Definition von struktureller Stabilität), sind in der Phase der Instabilität entscheidend; sie werden in dieser Phase extrem verstärkt, so daß kleine Ursachen riesige Effekte haben können. Dieses Phänomen liegt der thermodynamischen Strukturbildungstheorie von Prigogine (vgl. Prigogine/Allen 1982) und auch Hakens Synergetik (Haken 1983A) zu Grunde.

Ich will im folgenden eine spezielle synergetische Modellkonzeption, die von Weidlich (1972) sowie Weidlich/Haag (1980 und 1983) entwickelt wurde, auf unser Problem anwenden, um so die Modellbildungsmöglichkeiten der Synergetik in diesem Bereich zu beleuchten.

\subsection{Kurze Skizze des synergetischen Instrumentariums: die Master-Gleichung}

Eine ausführliche Darleguing der mathematischen Grundlagen des Verfahrens findet sich in Haken (1977: 75-96, besonders 75ff.). Die Grundideen sind informell in Haken (1983E: Kapitel 13: „Sind Revolutionen vorhersagbar?“) beschrieben. Wir beschränken uns auf eine halbformale Darstellung, welche die Struktur des formalen Modells nur skizziert.

Der einfachste Fall eines Zufallprozesses ist eine Partikel, welche sich in jedem Zeittakt genau einen Schritt auf einer Kette von Punkten bewegt. Mögliche Prozeßverläufe sind in Abb. 7 (vgl. Haken 1977: 79) angegeben. 


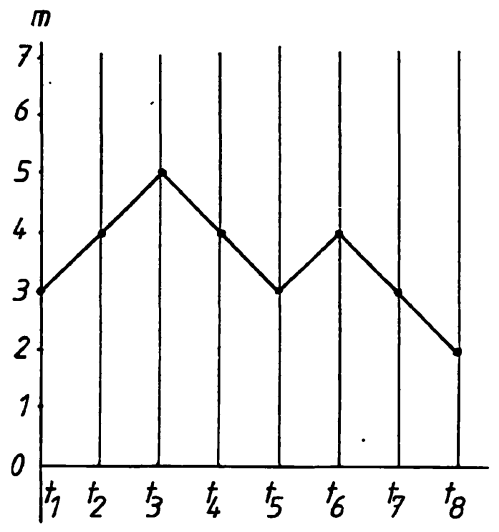

Abb. 7

Wenn wir eine Gesamtheit von Partikeln betrachten, können wir nicht nur das mittlere Verhalten und die Trends bzw. die Fluktuationen feststellen, wir können larüber hinaus gehen und kooperative Effekte untersuchen, d. h. uns fragen, wie lie Bewegung der einzelnen Partikel von der Bewegung anderer Partikeln ablängt.

Besonders einfache kooperative Effekte, die uns als anschauliche Bezugssunkte dienen mögen, finden wir beim Ferromagneten. Erhitzt man einen MagJeten über eine bestimmte kritische Temperatur Tc, so verliert er seinen Magneismus. Die zuvor geordneten und parallel ausgerichteten Eisenatome verlieren jie gemeinsame Ausrichtung. Dieser Zustand ist in Abb. 8 links dargestellt. Die kollektive Anordnung kann aber wieder hergestellt werden, indem der Ferromagnet auf eine Temperatur unterhalb der Schwelle Tc abgekühlt wird.

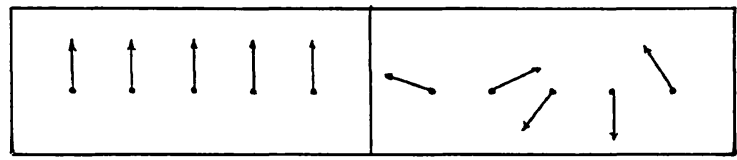

$A b b .8$

Das physikalische Modell mit spontaner Selbstorganisation kann zur approkimativen Simulation von kollektiven Prozessen in den weitaus komplexeren jystemen in der Gesellschaft benützt werden. Weidlich (1972) schlägt in dieser Linie ein Modell für die Migration in einer Stadt vor. Wir wollen das Modell- 
spicl von Weidlich (1972) und Weidlich/Haag $(1980 ; 1983)$ dazu benutzen, um : die Verwendbarkcit synergetischer Methoden in der Soziolinguistik exemplarisch aufzuzcigen. Wir gehen zuerst von der schrittweisen Bewegung der Partikel aus. In dicsem Fall ist die Wahrscheinlichkeit, daß sich die Partikel nach $\mathbf{n}+1$ Schritten im Zustand $m$ befindet:

$$
\mathrm{P}(\mathrm{m} ; \mathrm{n}+1)
$$

Bevor die Partikel im Zustand $m$ war, war sie wegen der schrittweisen Bewegungen entweder in $m+1$ oder in $m-1$ mit den entsprechenden Wahrscheinlichkeiten:

$$
w(m, m-1)=w(m, m+1)=1 / 2
$$

Die Übergangswahrscheinlichkeit von einem Zustand $m^{\prime}$ nach $n$ Schritten zu einem Zustand $m$ nach $n+1$ Schritten beträgt:

$$
P\left(m, n+1 ; m^{\prime}, n\right)=w\left(m, m^{\prime}\right) P\left(m^{\prime} ; n\right)
$$

D.h., die Grundwahrscheinlichkeit $P$, überhaupt nach $n$ Schritten in $\mathrm{m}^{\prime}$ zu sein, multipliziert mit der Wahrscheinlichkeit eines Übergangs von $\mathrm{m}^{\prime}$ nach $\mathrm{m}$.

Wir können bei Einführung einer Schrittdauer den Prozeß auch in Abhängigkeit von der Zeit formulieren (Zeit $t=$ Anzahl der Schritte $n \cdot$ Schrittdauer $\tau$ ), sc $\mathrm{da}$ gilt:

$$
\tilde{P}(m, t)=P(m ; n) \equiv P(m ; t / \tau)
$$

Die fundamentale Master-Gleichung beschreibt die Veränderung der Wahrscheinlichkeitsverteilung $\widetilde{P}(m, t)$ in der Abhängigkeit von der Zeit

$$
\frac{\mathrm{d} \tilde{\mathrm{P}}(\mathrm{m}, \mathrm{t})}{\mathrm{dt}}
$$

Entsprechend ist eine zeitabhängige Übergangswahrscheinlichkeit $\tilde{w}$ zu definieren. Global läßt sich die Veränderungsrate auf vier Faktoren zurückführen:

(a) Bewegungen zum Zustand $m$, entweder von $m+1$ oder von $m-1$ ausgehend;

(b) Bewegungen weg von $\mathrm{m}$; also nach $\mathrm{m}+1$ oder $\mathrm{m}-1$.

Diese sind jeweils durch das Produkt der Wahrscheinlichkeiten der Ausgangszustände und der Übergangswahrscheinlichkeiten bestimmt (vgl. (6)). 


$$
\begin{aligned}
& \text { (9) } \frac{\mathrm{d} \tilde{\mathrm{P}}}{\mathrm{dt}}=\begin{array}{rr}
\tilde{\mathrm{w}}(\mathrm{m}, \mathrm{m}-1) & \tilde{\mathbf{P}}(\mathrm{m}-1, \mathrm{t}) \\
+\tilde{\mathrm{w}}(\mathrm{m}, \mathrm{m}+1) & \tilde{\mathrm{P}}(\mathrm{m}+1, \mathrm{t})
\end{array} \\
& -\tilde{w}(m-1, m) \quad \tilde{P}(m, t) \\
& -\tilde{w}(m+1, m) \quad \tilde{P}(m, t)
\end{aligned}
$$

Dies ist die einfachste Form der Master-Gleichung.

\subsection{Ein einfaches Modell für den Prozeß des Sprachwechsels}

Wir betrachten zuerst eine homogene soziale Gruppe mit zwei möglichen $\mathrm{Zu}$ ständen:

$\mathrm{N}=$ Wahl des Niederdeutschen als Sprache

$\mathrm{H}=$ Wahl des Hochdeutschen als Sprache.

Die Zahl der Personen, welche sich für Niederdeutsch entscheiden, sei $\mathbf{n}_{\mathbf{N}}$; die Zahl derjenigen, welche das Hochdeutsche wählen, sei $n_{H}$. Es gilt:

$$
\mathrm{n}_{\mathrm{N}}+\mathrm{n}_{\mathrm{H}}=\mathrm{n} \quad(=\text { Gesamtpopulation })
$$

Die relativen Anteile der beiden Sprachen an der Population sind dann:

$\mathrm{x}_{\mathrm{N}}=\mathrm{n}_{\mathrm{N}} / \mathbf{n}: \mathrm{x}_{\mathrm{H}}=\mathrm{n}_{\mathrm{H}} / \mathrm{n}$.

Wir können die Dynamik der Veränderung in der Population als Übergänge ' $z$ wischen $\mathrm{N}$ und $\mathrm{H}$ oder quantitativ als Veränderung der Stärke der jeweiligen Gruppen $n_{N}, n_{H}$ beschreiben; dabei sind die Übergangswahrscheinlichkeiten:

$P_{\mathbf{N} \rightarrow \mathrm{H}}:$ Niederdeutsch $\rightarrow$ Hochdeutsch

$P_{\mathbf{H} \rightarrow \mathbf{N}}:$ Hochdeutsch $\rightarrow$ Niederdeutsch

Die speziellen Modellbildungskontexte legen es nahe, folgende Parameter einrnführen, welche die Übergangswahrscheinlichkeit beeinflussen:

1) individuelle Präferenzen für das Niederdeutsche (positiv) oder das Hochdeutsche (negativ): $P$

2) individuelle Anpassungstendenzen an das Wahlverhalten der sozialen Umgebung: A

3) soziales Klima, d.h. Freiheit des einzelnen oder soziale Kontrolle durch die Gesamtgesellschaft: $\mathbf{K}$

tlle drei Parameter sind sowohl in Weidlichs Migrationsmodell als auch in unseem Anwendungsfeld sinnvoll. Unter diesen Bedingungen (die bei einer exakten 
Simulation zu überprüfen wären) ergibt sich eine Parallelität zum Ising-Modell des Ferromagnetismus, wobci positive und negative Polung der Sprachwahl in unserem Modell entspricht. Wir können deshalb die Dynamik exemplarisch an der Simulation des Ising-Modells untersuchen. Die drei Parameter: P (Präferenz), A (Anpassung) und $K$ (soziales Klima) sind verknüpft, so da $B$ wir zwei Grundparameter festlegen können.

$$
\mathrm{k}=\frac{\mathrm{A}}{\mathrm{K}} ; \mathrm{h}=\frac{\mathrm{P}}{\mathrm{K}}
$$

( $k$ = Anpassung relativ zum sozialen $\mathrm{Klima}, \mathrm{h}=$ Präferenz relativ zum sozialen Klima).

Die Dimensionen der Ergebnisdarstellung sind:

(a) Die stationäre Lösung des Gleichungssystems (vgl. die Master-Gleichung, die allerdings in diesem Falle erweitert und modifiziert wurde). $\mathrm{f}_{\mathrm{st}}(\mathrm{x})$

(b) Die zusammenfassende Variable $\mathrm{x}$. Sie wird wie folgt berechnet: $x=1 / 2\left(x_{N}-x_{H}\right)$

d.h. die Hälfte der Distanz zwischen den relativen Anteilen der beiden Sprachen.

Wir zeigen (in Anschluß an Weidlich 1972: 57f.) vier charakteristische Situationen in der Simulation des Systems $(n=100)$.

(a) Weder Anpassung noch Präferenz $(\mathrm{k}=0, \mathrm{~h}=0)$

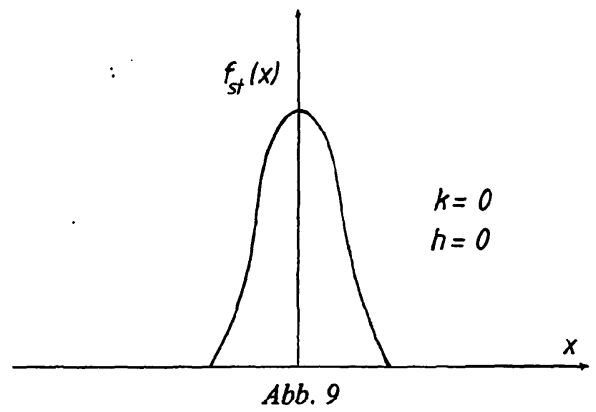


Die Wechsel bezüglich der beiden Alternativen $\mathrm{N}, \mathrm{H}$ heben sich gegenseitig auf, die Situation ist stabil ohne Strukturveränderung.

(b) Mittlere Anpassung, keine Präferenz

$$
\left(k=k_{0}=2 ; h=0\right)
$$

An dieser Grenze der stabilen Phase sehen wir ein breites Spektrum von Abweichungen mit geringer Richtungstendenz (shift), aber hoher Fluktuation.

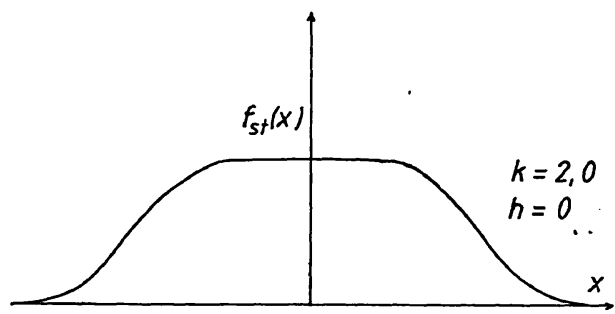

Abb. 10

(c) Jenseits des kritischen Wertes des Anpassungsparameters $\left(k_{0}=2\right)$ kommt es zur Polarität. Wir betrachten den Fall $k=2,5 ; \mathrm{h}=0$.

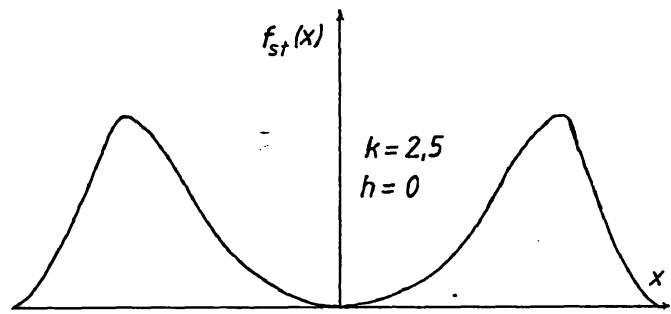

Abb. 11

Es gibt Verānderungsflüsse in beide Richtungen, also zur Sprache $\mathrm{N}$ und $\mathrm{H}$. Allerdings sind wir von einer neutralen Präferenz ausgegangen $(h=0)$. Wenn wir die Präferenz nur geringfügig verändern, kippt die Situation auf die Seite der I Präferenz, die Symmetrie in Abb.11 wird gebrochen.

(d) Anpassung jenseits des kritischen Wertes und leichte Präferenz für eine Sprache $(k=2,5 ; k=0,01)$ 


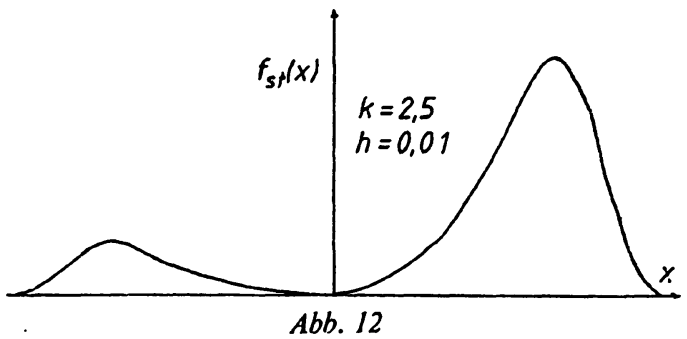

Die Situation (d) ist für unseren Fall deshalb von besonderer Relevanz, weil sie zeigt, da $B$ bei verstärkter, individueller Anpassung in einer generell sozial gelockerten Situation $\left(\frac{\mathrm{A}}{\mathrm{K}}>0\right)$ eine durch verstärkte Fluktuationen gesteuerte Polarisierung ausgelöst wird; diese ist aber in instabiler Symmetrie, so da $B$ die geringste Verschiebung der Präferenz (etwa die Hoffnung, mit Hochdeutsch den sozialen Aufstieg, insbesondere vermittelt durch die Schule für sich oder seine Kinder zu ermöglichen, oder die Einheitseuphorie der 70er und 80er Jahre) bereits die Situation kippen läßt und so in kurzer Zeit zu einer radikalen Verānderung der gesprochenen Stadtsprache führt.

\subsection{Ein differenzierteres Modell}

In Weidlich/Haag (1980 und 1983: Kap. 4) wird ein erweitertes Modell mit ähnlichen Parametern, aber zwei Startpopulationen $P_{u}, P_{v}$ errechnet. Der Faktor $k$ im Modell des vorherigen Abschnittes wird entsprechend aufgespalten in: $\left(k_{u}, k_{v}\right)$ und $\left(\mathrm{g}_{\mathrm{u}}, \mathrm{g}_{\mathrm{v}}\right)$.

(a) $k_{u}, k_{v}$ : die Bereitschaft der Gruppe $P_{u}$ bzw. $P_{v}$, dieselbe Wahl zu treffen: interne Sympathie;

(b) $g_{u}, g_{v}$ : die Bereitschaft, mit der anderen Population eine gemeinsame Wahl zu treffen: externe Sympathie.

Wir schildern kurz die wesentlichen Veränderungen im Modell: Die beiden Populationen $P_{u}$ und $P_{v}$ verändern ihre Wahl bezüglich der Alternativen $N$ $=$ Niederdeutsch und $\mathrm{H}=$ Hochdeutsch. Als jeweilige Quoten erhalten wir:

$m_{N}, m_{H}:\left(m_{N}+m_{H}\right)=2 \bar{m}:$ Gesamtheit der Sprecher in $P_{u}$ $\mathrm{n}_{\mathrm{N}}, \mathrm{n}_{\mathrm{H}}:\left(\mathrm{n}_{\mathrm{N}}+\mathrm{n}_{\mathrm{H}}\right)=2 \overline{\mathrm{n}}:$ Gesamtheit der Sprecher in $\mathrm{P}_{\mathrm{v}}$

Wir können wegen dieser Abhängigkeiten von zwei Variablen $m$ und $\mathbf{n}$ ausgehen (den jeweiligen Abweichungen von den Mittelwerten $\overline{\mathrm{m}}$ und $\overline{\mathbf{n}}$ ). 
$\mathrm{m}_{\mathrm{N}}=\overline{\mathrm{m}}+\mathrm{m}, \quad \mathrm{m}_{\mathrm{H}}=\overline{\mathrm{m}}-\mathrm{m}$

$\mathrm{n}_{\mathrm{N}}=\overline{\mathrm{n}}+\mathrm{n}, \quad \mathrm{n}_{\mathrm{H}}=\overline{\mathrm{m}}-\mathrm{n}$

Anstelle der Abweichungen vom Mittelwert: $m$ und $n$, können wir wie im vorherigen Abschnitt relative Abweichungen $x$ und $y$ definieren.

$\mathrm{x}=\frac{\mathrm{m}}{\overline{\mathrm{m}}} ; \quad \mathrm{y}=\frac{\mathrm{n}}{\overline{\mathrm{n}}}$ mit: $\quad-1 \leq \mathrm{x}, \mathrm{y} \leq+1$

Statt der zweidimensionalen Bilder im vorherigen Abschnitt erhalten wir nun dreidimensionale Bilder mit den Variablen $x$, $y$ und den stationären Lösungen der Master-Gleichung: $p_{s t}(x, y)$ als Ordinate.

In unserer Anwendung könnten die Populationen $P_{u}, P_{v} z$. B. als zwei soziale Schichten oder als Stadtkern vs. Stadtperipherie oder als alteingesessene vs. zugewanderte Einwohnerschaft aufgefaßt werden. Wir wollen nur grob betrachten, welche Folgen die Interaktion von zwei Populationen hat:

(a) Bei neutraler Präferenz: $h_{u}, h_{v}=0$; schwacher interner Sympathie: $k_{u}=k_{v}$ $=0,2$ und externer Sympathie: $g_{u}=g_{v}=0,5$ tendiert die Dynamik auch bei inhomogenen Ausgangssituationen zu einer homogenen Verteilung der Gruppen über die Sprachen N und H. Abb. 13 zeigt diese Situation (vgl. Weidlich/Haag 1983: 104).

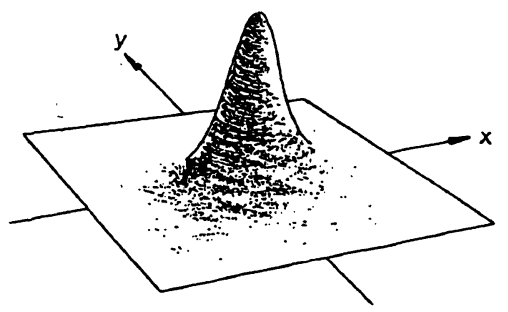

Abb. 13

(b) Bei schwacher interner Sympathie: $k_{u}=k_{v}=0,5$ und stärkerer externer Sympathie: $g_{u}=g_{v}=1,0$ und bei neutraler Präferenz kommt es zu einer Wanderung in Richtung auf die Majorität der anderen Gruppe. In Abhängigkeit vom Ausgangspunkt nehmen beide Gruppen dieselbe Wahl vor. Bei negativer externer Sympathie, d.h. bei Antipathie, kommt es zu Absonderung der beiden Populationen bez. der zu treffenden Wahl.

In unserer Anwendung ergäbe dies: 
(aa) Einheitliche Sprachwahl (entweder Nicderdeutsch oder Hochdeutsch) als stabiler Endzustand.

(bb) Konträre Sprachwahl als stabiler Endzustand. Es entstehen harte Sprachfronten oder gar "Sprachghettos".

Abb. 14 zeigt dic Situationen in (aa) (Weidlich/Haag 1983: 105) die Situation (bb) entsprächc einer Spicgelung von Abb.14 an der x-Achse.

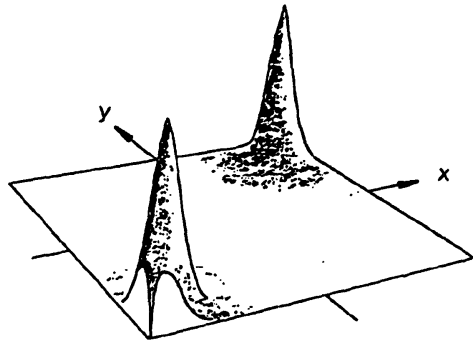

Abb. 14

(c) Die Situation in (aa) wird asymmetrisch, sobald eine schwache Präferenz für eine der Sprachen aufkommt; $d$. h., wenn starke interne und externe Sympathien existieren und somit die kooperativen Effekte stark sind, hat bereits eine schwache Präferenz eine starke Dynamik hin zur präferierten Lösung zur Folge.

Bis jetzt waren die Parameter immer gleichgerichtet und auch gleich stark in beiden Populationen. Für unsere Anwendung sind die Fälle besonders interessant, in denen die externe Sympathie asymmetrisch ist. So wollen etwa stadtperiphere Gruppen gerne ,städtisch“ sein, aber die Stadtbevölkerung hat kein Interesse ,ländlich“" zu werden; Personen aus der Unterschicht oder unteren Mittelschicht streben eventuell den Lebensstil der oberen Mittelschicht an, ohne da $§$ die Personen der oberen Mittelschicht ihrerseits den Lebensstil der Unterschicht anstreben. Die Ergebnisse der Modellsimulationen in Weidlich/Haag 1983: 108-111) lassen vermuten, da $B$ in diesen Fällen die interne Sympathie entscheidet (bei neutraler Präferenz). Ist diese schwach, so erhalten wir eine homogene Zweisprachigkeit, wie in (a). Bei stärkerer interner Sympathie (und asymmetrischer externer Sympathie) kommt es zu sehr starken Fluktuationen, wobei ganze Gruppen aus $P_{u}$ oder $P_{v}$ die Sprache wechseln; es entsteht eine sehr instabile Situation. Bei extremer interner Sympathie kommt es zu einer zyklischen Verdrängung insofern, als die Gruppe, welche positive externe Sympathien hat, sich ihrer Zielgruppe annähert; diese sich aber wegbewegt, so daß im Modell ein 
Grenzzyklusverhalten eintritt. In unserem Anwendungsbereich wäre das etwa gegeben, wenn die eine Population z. B. das Niederdeutsche aufgibt, um sich der sozial dominanten Gruppe anzupassen; diese würde im Gegenzug ein feineres Hochdeutsch (oder eine andere Sprachvariante) wählen, um den Abstand wiederherzustellen.

An diesen Beispielen lassen sich einerseits die Grenzen der Übertragung der Vorlage von Weidlich/Haag (1983) auf unsere Problemstellung deutlich machen. andererseits kann ein entsprechender Proze $B$ durchaus plausibel sein, wenn man bedenkt, daß die Sprachen sich auch unabhängig von den betrachteten Einstellungen und Bewertungen verändern und somit immer wieder neue Varianten verfügbar sind. Eine Kleingesellschaft, welche nicht wie die Stadt Bremen in überregionale Kommunikationsnetze eingebettet ist und auch nicht in der Form einer tradierten und durch Institutionen kontrollierten Schriftsprache einer höheren Kontrolle ausgesetzt ist, könnte durchaus eine Spirale von Sprachformen durchlaufen, wobei die prestigeorientierte Gruppe Sprachformen übernimmt, welche die Prestigegruppe ihrerseits ablegt, um den Abstand zu erhalten. In der heutigen Gesellschaft sind solche Prozesse jedoch wesentlich vielschichtiger. Genau gesehen gibt es nicht nur zwei zu wählende Varianten, sondern eine ganze Landschaft koexistierender und konkurrierender Varianten, welche zudem einer internen, zeitabhängigen Entwicklung unterworfen sind.

\section{Ausblick}

Diese Studie hatte zum Ziel, an einem ausgewählten und empirisch gut zugänglichen Beispiel zu zeigen, wie synergetische Modellkonzepte in die Linguistik Eingang finden können. Wirklich spannend wird die Anwendung aber erst, wenn sich die Beobachtungen zumindest approximativ quantifizieren lassen, so da $B$ eine feinere Modellanpassung möglich wird. Dies würde außerdem eine breitere Theoriebildung ermöglichen, in der andere soziale und ökonomische Prozesse ebenfalls als dynamisches System rekonstruiert würden. Die Sprachdynamik I wäre dann in ihrer konkreten Ankopplung an soziale und ökonomische Prozesse erfaßbar. Damit käme eine theoretische Soziolinguistik in Reichweite und das derzeitige Stadium vager Korrelationshypothesen könnte endlich überwunden werden.

\section{Literaturnachweis}

[Allen 1982] Allen, Peter: Self-organisation in the urban system. - In: (Schieve und Allen 1982), S. 132-158.

[Allen/Sanglier 1981] Allen, Peter/Sanglier, Michèle: Order by fluctuation and the urban system. - In: Autopoiesis, Dissipative Structures, and Spontaneous Social Orders. Ed. by Milan Zeleny. - Boulder: Westview Press 1981. S. 109-131. 
[Binder 1982] Binder, Heinrich: Der Jeini von Bremen. (1. Auflagc, 1923). - Bremen: Schünemann 1982.

[Brüggebors 1966] Brüggebors, Traute: Dic Verbreitung des Plattdeutschen an Bremer Ifauptschulen. - In: Mitteilungen des Vereins für Niedersächsisches Volkstum e. V. 41 (1966), 64-69.

[Bunning 1935] Bunning, Hcinrich: Studien zur Geschichte der Bremischen Mundart (seit dem Untergang der millelniederdeutschen Schriftsprache). - In: Jahrbuch des Vereins für nicderdeutsche Sprachforschung 60/61 (1935), 4/35, 63-147.

[Carcy 1858/59] Carey, Henry Charles: Principles of social science. - Philadelphia: Lippinscolt $1858 / 59$.

[Coffey 1981] Coffey, William James: Geography. Towards a general spatial systems approach. - London: Methuen 1981.

[Cordes/Möhn 1980] Handbuch zur niederdeutschen Sprach- und Literaturwissenschaft. Hrsg. von Gerhard Cordes und Dieter Möhn. - Berlin: Schmidt 1980.

[Droste 1913] Droste, Georg: Otıjen Alldag un sien Kaperstreiche (Bd 1); Ottjen Alldag un sien Lehrtied (Bd 2). (8. bzw. 4. Auflage). - Bremen: Schünemann 1913.

[Gabriclsson 1933] Gabriclsson, Artur: Das Eindringen der hochdeutschen Sprache in die Schulen Niederdeutschlands in 16. und 17. Jahrhundert. - In: Jahrbuch des Vereins fur niederdeutsche Sprachforschung 58/59 (1933), 1-79.

[Gabrielsson 1980] -: Die Verdrängung der mittelniederdeutschen durch die neuhochdeutsche Schriftsprache. - In: (Cordes und Möhn 1980), S.119-153.

[GETAS 1979] GETAS-Institut Bremen: Ergebnisse einer Repräsentativ-Erhebung. Im Auftrag des Instituts für niederdeutsche Sprache. [Unveröffentl. Papier, Bremen: 1979].

[Goossens 1973] Niederdeutsch. Sprache und Literatur. Eine Einführung. Hrsg. von Jan Goossens. Bd1: Sprache. - Neumünster: Wachholtz 1973.

[Haken 1977] Haken, Hermann: Synergetics. An introduction. - Berlin: Springer 1977.

[Haken 1983A] -: Advanced synergetics. Instability hierarchies of self-organizing sy'stems and devices. - Berlin: Springer 1983.

[Haken 1983 E] -: Erfolgsgeheimnisse der Natur. Synergetik: Die Lehre vom Zusammenwirken. - Stuttgart: Deutsche Verlagsanstalt 1983.

[Hard 1983] Hard, Gerhard: Ein geographisches Simulationsmodell für die rheinische Sprachgeschichte. - In: Festschrift Matthias Zender. Hrsg. von E. Ennen/G. Wiegelmann. - Bonn: Röhrscheid 1983. S. 25-28.

[Hoffmeister 1977] Hoffmeister, Walter: Sprachwechsel in Ost-Lothringen. - Wiesbaden: Steiner 1977.

[Janßen 1943] Janßen, Hans: Leben und Macht der Mundart in Niedersachsen. - Oldenburg 1943. (= Veröffentlichungen des Provinzial-Instituts für Landesplanung und niedersächsische Landesforschung Hannover-Göttingen. 14).

[Kiefer 1966] Kiefer, Klaus: Die Diffusion von Neuerungen. - Tübingen: Mohr 1967.

[Kippenberg 1942] Kippenberg, Anton: Geschichten aus einer alten Hansestadt. - Leipzig: Insel-Verlag 1942.

[Kohlheim 1981] Kohlheim, Volker: Diffusionstheoretische Aspekte spätmittelalterlicher Anthroponymie. - In: Deutsch-Slawische Namenforschung. Hrsg. von H.-B. Harder. Marburg: Herder-Institut 1981. S.137-157.

[Labov 1966] Labov, William: The social stratification of English in New York City. Washington: Center of Applied Linguistics 1966.

[Matthes u. a. 1981] Biographie in handlungswissenschaftlicher Perspektive. Hrsg. von Joachim Matthes, Arno Pfeiffenberger und Manfred Stosberg. - Nürnberg: Verlag der Nürnberger Forschungsvereinigung e.V. 1981.

[Möhn 1973] Möhn, Dieter: Deutsche Stadt und niederdeutsche Sprache. - In: Niederdeutsches Jahrbuch 96 (1973), 111-126. 
[Möhn 1980] -: Geschichte der neuniederdeutschen Mundarten. - In: (Cordes und Möhn 1980), S.155-181.

[Mottron/Wildgen 1986] Mottron, Laurent/Wildgen, Wolfgang: Dynamische Sprachtheorie. Dynamische Modelle für die Sprachstruktur, den frühen Spracherwerb und seine Pathologien. - Bochum: Brockmeyer 1986.

[Niethammer 1980] Lebenserfahrung und kollektives Gedächtnis. Die Praxis der ,Oral History.". Hrsg. von Lutz Niethammer. - Frankfurt 1980.

[Peters 1973] Peters, Robert: Mittelniederdeutsche Sprache. - In: (Goossens 1973), S. 66115.

[Prigogine/Allen 1982] Prigogine, llya und Allen, Peter M.: The challenge of complexity.In: (Schieve und Allen 1982), S.3-39.

[Prigogine/Stengers 1983] Prigogine, Ilya/Stengers, Isabelle: Dialog mit der Natur. Neue Wege naturwissenschaftlichen Denkens. - München: Piper 1983.

[Ravenstein 1885] Ravenstein, E. G.: The laws of migration. - In: Journal of the Royal Statistical Society 48 (1885), 167-235.

[Röhrs 1976] Röhrs. Gustav: Bremen-Ost, mit Heini, Willi, Didi-Kalli, Jan und Fidi. Erlebtes und Erlauschtes eines Bremer Jungen. (2. erweiterte Auflage). - Bremen 1976.

[Schieve/Allen 1982] Self-organisation and dissipative structures. Applications in the physical and social sciences. Ed. by William C. Schieve and Peter Allen. - Austin: University of Texas Press 1982.

[Schwarzwälder 1976] Schwarzwälder, Herbert: Geschichte der Freien Hansestadt Bremen. Bd II: Von 1810 bis zum Ersten Weltkrieg 〈1918〉. - Bremen: Röver 1976.

[Sodmann 1973] Sodmann, Timothy: Der Untergang des Mittelniederdeutschen als Schriftsprache. - In: (Goossens 1973), S. 116-129.

[Trudgill 1974] Trudgill, Peter M.: Linguistic change and diffusion. Description and explanation in sociolinguistic dialectgeography. -In: Language and Society 3(1974), 215-246.

[Weidlich 1972] Weidlich, Wolfgang: The use of statistical models in sociology. - In: Collective Phenomena 1 (1972), 51-59.

[Weidlich/Haag 1980] Weidlich, Wolfgang/Haag, Günter: Migration behavior of mixed population in a town. - In: Collective Phenomena 3 (1980), 89-102.

[Weidlich/Haag 1983]-: Concepts and models of a quantitative sociology. The dynamics of interacting populations. - Berlin: Springer 1983.

[Wildgen 1982] Wildgen, Wolfgang: Catastrophe Theoretic semantics. An application and elaboration of René Thom's theory. - Amsterdam: Benjamins 1982.

[ [Wildgen 1984G] -: Goethe als Wegbereiter einer universalen Morphologie. L.A.U.T. Preprint. Series A, Nr.125. - Trier 1984.

[Wildgen 1984S] -: Sprache als selbstorganisierendes System. L.A.U.T. Preprint, Series A, Nr.126. - Trier 1984.

$\therefore$; [Wildgen 1985] -: Archetypensemantik. Grundlagen für eine Dynamische Semantik auf der Basis der Katastrophentheorie. - Tübingen: Narr 1985.

! [Zeemann 1982] Zeemann, Christopher: Bibliography on Catastrophe Theory. - Warwick 1982. [Mimeo].

Eingereicht am 27.3.1985 